\title{
Completeness of Sequence Spaces Generated by an Orlicz Function
}

\section{Kelengkapan Ruang Barisan yang terdefinisi oleh Fungsi Orlicz}

\author{
Nur Khusnussa'adah ${ }^{1, *}$, Supama ${ }^{2}$ \\ ${ }^{1}$ Departemen Matematika FMIPA UGM, Yogyakarta \\ ${ }^{2}$ Departemen Matematika FMIPA UGM, Yogyakarta \\ *nur-khusnus@ugm.ac.id
}

\begin{abstract}
In this paper, we discuss about completeness property of Orlicz sequence space as one of sequence spaces defined by an Orlicz function. Orlicz sequence space is generalization of $p$-summable sequence space, for every $p \geq 1$ which is also an Orlicz sequence space. Based on the property of convergence sequence on norm space, we define $\Phi$-convergence sequence on Orlicz sequence space. Moreover, we define $\Phi^{-}$ Cauchy sequence and $\Phi$-complete on Orlicz sequence space. In this paper, we show the relationship between the (ordinary) convergent sequence, $\Phi$-convergent and $\Phi$-Cauchy sequence. Finally, it will also be shown that Orlicz sequence space is Banach space and $\Phi$-complete space.
\end{abstract}

Keywords: Convergence Sequence; Orlicz Function; Sequence Space; Orlicz Sequence Space

\begin{abstract}
Abstrak
Dalam paper ini, dibahas tentang sifat kelengkapan dalam ruang barisan Orlicz, yaitu suatu ruang barisan yang terdefinisi oleh fungsi Orlicz. Ruang barisan Orlicz merupakan generalisasi dari ruang barisan summable- $p$, untuk setiap $p \geq 1$ yang juga merupakan ruang barisan Orlicz. Berdasarkan sifat kekonvergenan barisan pada ruang bernorma, didefinisikan sifat barisan $\Phi$-konvergen pada ruang ruang barisan Orlicz. Lebih lanjut, didefinisikan juga sifat barisan $\Phi$-Cauchy dan $\Phi$-lengkap pada ruang tersebut. Pada paper ini, akan ditunjukkan hubungan antara barisan konvergen (biasa), $\Phi$-konvergen dan barisan $\Phi$-Cauchy. Pada bagian akhir, akan ditunjukkan juga bahwa ruang barisan Orlicz ruang Banach dan ruang $\Phi$-lengkap.
\end{abstract}

Kata Kunci: Barisan Konvergen, Fungsi Orlicz, Ruang Barisan, Ruang Barisan Orlicz

\section{Pendahuluan}

Ruang Orlicz pertama kali diperkenalkan pada tahun 1931 oleh Z.W. Birnbaum dan Wladyslaw Orlicz. Selanjutnya, pada tahun 1932, Orlicz mengembangkan sendiri ruang tersebut sebagai perluasan dari ruang Lebesgue $L_{p}$. Ruang barisan Orlicz adalah kasus khusus dari ruang Orlicz dan merupakan generalisasi ruang barisan summable- $p \ell_{p}$, untuk setiap $p \geq 1$. 
Pengkajian ruang barisan Orlicz tersebut mulai diperkenalkan oleh Lindenstrauss bersama Tzafriri (1973) dan Lindenberg (1973). Lindenstrauss dan Tzafriri terinspirasi untuk mengkonstruksi suatu ruang barisan yang dibangkitkan oleh fungsi Orlicz. Ruang barisan tersebut merupakan ruang Banach yang selanjutnya disebut ruang barisan Orlicz. Ide pengkonstruksian ruang barisan yang dibangkitkan oleh fungsi Orlicz seperti yang dilakukan oleh Lindenstrauss dan Tzafriri ternyata telah menginspirasi peneliti-peneliti lain, di antaranya Kamthan dan Gupta (1981), Alexopoulos (2004), E. Savas dan R. Savas (2004), Khan (2008), dan Kolk (2011).

Berdasarkan sifat kekonvergenan barisan dan kelengkapan ruang bernorma yang dijelaskan oleh Kreyszig (1978), selanjutnya didefinisikan beberapa konsep, di antaranya $\Phi$ konvergen dan $\Phi$-lengkap di dalam ruang barisan Orlicz. Pada paper ini dibahas tentang sifat-sifat dalam ruang barisan Orlicz, hubungan $\Phi$-konvergen dengan konvergen (biasa), dan sifat $\Phi$ lengkap. Topik-topik tersebut menarik untuk dikaji, terutama karena aplikasinya baik di bidang matematika analisis sendiri seperti yang dituliskan oleh Rao dan Ren (2002), maupun aplikasi di bidang lain yang terkait. Aplikasi yang dimaksud antara lain approximation theory, operator nonlinear, analisis Fourier, persamaan diferensial nonlinear, persamaan integral, dan analisis stokastik.

\section{Metode Penelitian}

Terlebih dahulu dikaji materimateri dasar yang nantinya digunakan sebagai acuan untuk penelitian inti, antara lain tentang kekonvergenan dan kelengkapan suatu barisan dalam ruang bernorma. Selanjutnya, didefinisikan fungsi Orlicz $\Phi$ yang memenuhi syaratsyarat tertentu serta $\Psi$ sebagai komplemen fungsi Orlicz $\Phi$. Fungsi Orlicz $\Phi$ membangkitkan ruang barisan baru yang dinamakan $X_{\Phi}$. Ruang $X_{\Phi}$ dibangkitkan oleh fungsi Orlicz $\Phi$ dengan berbagai macam cara. Salah satu contoh dari ruang $X_{\Phi}$ adalah ruang $\ell_{\Phi}$, yang selanjutnya disebut sebagai ruang barisan Orlicz. Di dalam ruang $\ell_{\Phi}$ dipelajari sifat-sifat yang berlaku, diantaranya tentang kekonvergenan dan kelengkapan suatu barisan.

\section{Hasil dan Pembahasan}

Pada bagian ini terlebih dahulu diperkenalkan tentang fungsi Orlicz, norma Luxemburg, norma Orlicz, dan 
ruang barisan Orlicz serta sifat-sifat yang berlaku di dalamnya. Pengertian fungsi Orlicz diberikan pada definisi berikut.

Definisi 1. Fungsi $\Phi: \mathfrak{R} \rightarrow \mathfrak{R}^{+}$disebut fungsi Orlicz (Orlicz function) jika:

i. $\Phi$ kontinu pada $\mathfrak{R}$

ii. $\Phi(-x)=\Phi(x)$, untuk setiap $x \in \mathfrak{R}$

iii. Ф naik pada $\mathfrak{R}^{+}$

iv. $\Phi(x)=0$ jika dan hanya jika $x=0$.

Untuk lebih memahami definisi tersebut, berikut ini diberikan beberapa contoh fungsi Orlicz.

Contoh 1. Untuk $p \geq 1$, fungsi $\Psi: \Re \rightarrow \mathfrak{R}^{+}$dengan $\Psi(x)=|x|^{p}$, untuk setiap $x \in \mathfrak{R}$ merupakan fungsi Orlicz, sebab memenuhi sifat-sifat berikut:

i. Bentuk fungsi $\Psi$ sebagai komposisi dari dua fungsi $\mu$ dan $\eta$ , yaitu $\Psi=\mu \circ \eta$ dengan $\mu(x)=x^{p}$ dan $\eta(x)=|x|$, untuk setiap $x \in \mathfrak{R}$. Karena fungsi $\mu, \eta$ masing-masing kontinu pada $\mathfrak{R}$, maka komposisi dua fungsi tersebut juga kontinu pada $\mathfrak{R}$.

ii. Untuk setiap $x \in \mathfrak{R}$, berlaku $\Psi(-x)=|-x|^{p}=|-1|^{p}|x|^{p}=|x|^{p}=\Psi(x)$ iii. Karena $\Psi^{\prime}(x)=p x^{p-1}>0$, untuk $x>0$, maka $\Psi$ fungsi naik pada $\mathfrak{R}^{+}$.

iv. Akan ditunjukkan

$\Psi(x)=0 \Leftrightarrow x=0$

.Jika $x=0$, maka $\Psi(0)=|0|^{p}=0$.

Jika $\Psi(x)=0$, berarti $|x|^{p}=0$, untuk $p \geq 1$ sehingga $x=0$.

Contoh 2. Diberikan fungsi $\Phi: \mathfrak{R} \rightarrow \mathfrak{R}^{+}$ dengan definisi $\Phi(x)=e^{|x|}-|x|-1$, untuk setiap $x \in \mathfrak{R}$. Fungsi $\Phi$ tersebut merupakan fungsi Orlicz, sebab memenuhi sifat-sifat berikut:

i. Bentuk fungsi $\Phi$ sebagai selisih dari tiga fungsi $\varphi, \mu$, dan $\eta$, yaitu $\Phi=\varphi-\mu-\eta \quad$ dengan $\varphi(x)=e^{|x|}$, $\mu(x)=|x|$, dan $\eta(x)=1$, untuk setiap $x \in \mathfrak{R}$. Karena fungsi $\varphi, \mu, \eta$ masing-masing kontinu pada $\mathfrak{R}$, maka $\Phi$ juga kontinu pada $\mathfrak{R}$.

ii. Untuk setiap $x \in \mathfrak{R}$, berlaku

$$
\begin{aligned}
& \Phi(-x)=e^{|-x|}-|-x|-1=e^{|(-1) x|}-|(-1) x|-1 \\
& =e^{|-1| x \mid}-|-1||x|-1 \\
& =e^{|x|}-|x|-1=\Phi(x) .
\end{aligned}
$$

iii. Karena $\Phi^{\prime}(x)=e^{x}-1>0$, untuk $x>0$, maka $\Phi$ fungsi naik pada $\mathfrak{R}^{+}$. 
iv. Akan ditunjukkan

$\Phi(x)=0 \Leftrightarrow x=0$.

Jika $x=0$, maka

$\Phi(0)=e^{|0|}-|0|-1=0$

Jika $\Phi(x)=0$, berarti

$e^{|x|}-|x|-1=0$. Andaikan $x \neq 0$.

Karena $\Phi$ naik pada $\mathfrak{R}^{+}$dan $\Phi$

genap, maka untuk $|x|>0$ berlaku

$\Phi(x)>\Phi(0)=0$. Kontradiksi.

Jadi, $x=0$.

Selanjutnya pengertian kondisi$\Delta_{2}$ dalam fungsi Orlicz diberikan pada definisi berikut.

Definisi 2. Fungsi $\Phi$ dikatakan memenuhi kondisi- $\Delta_{2}$, ditulis $\Phi \in \Delta_{2}$, jika terdapat bilangan real $K>0$ sehingga $\Phi(2 x) \leq K \Phi(x)$, untuk setiap $x \geq 0$.

Berikut ini diberikan contoh fungsi Orlicz yang memenuhi kondisi$\Delta_{2}$ dan tidak memenuhi kondisi- $\Delta_{2}$.

Contoh 3. Fungsi $\Psi$ yang didefinisikan pada Contoh 1 merupakan fungsi Orlicz yang memenuhi kondisi$\Delta_{2}$ karena untuk setiap $x \geq 0$ berlaku

$$
\Psi(2 x)=|2 x|^{p}=2^{p}|x|^{p}=2^{p} \Psi(x) .
$$

Jika diambil $K=2^{p}>0$ maka berlaku $\Psi(2 x) \leq K \Psi(x)$ untuk setiap $x \geq 0$.
Contoh 4. Fungsi $\Phi$ yang didefinisikan pada Contoh 2 merupakan fungsi Orlicz yang tidak memenuhi kondisi- $\Delta_{2}$.

Bukti: Diandaikan $\Phi$ memenuhi kondisi- $\Delta_{2}$, maka terdapat bilangan real $K>0$ sehingga untuk setiap $x \geq 0$ berlaku

$$
\begin{aligned}
& K\left(e^{x}-x-1\right)=K \Phi(x) \geq \Phi(2 x)=e^{2 x}-2 x-1 \\
& =\int_{0}^{2 x}\left(e^{p}-1\right) d p \\
& =\int_{0}^{x}\left(e^{p}-1\right) d p+\int_{x}^{2 x}\left(e^{p}-1\right) d p \\
& \geq \int_{x}^{2 x}\left(e^{p}-1\right) d p \geq \int_{x}^{2 x}\left(e^{x}-1\right) d p \\
& =\left.\left(e^{x}-1\right) p\right|_{x} ^{2 x} \\
& =x\left(e^{x}-1\right) .
\end{aligned}
$$

Karena untuk $x>0$ berlaku $e^{x}-x-1>0, \quad$ maka $\quad K \geq \frac{x\left(e^{x}-1\right)}{e^{x}-x-1}$, untuk setiap $x>0$. Akibatnya, jika diambil $x \rightarrow \infty$, maka

$$
\begin{aligned}
& K \geq \lim _{x \rightarrow \infty} \frac{x\left(e^{x}-1\right)}{e^{x}-x-1}=\lim _{x \rightarrow \infty} \frac{\left(e^{x}-1\right)+x e^{x}}{e^{x}-1} \\
& =\lim _{x \rightarrow \infty} \frac{e^{x}+e^{x}+x e^{x}}{e^{x}}=\lim _{x \rightarrow \infty} \frac{e^{x}(x+2)}{e^{x}} \\
& =\lim _{x \rightarrow \infty}(x+2)=\infty .
\end{aligned}
$$

Terjadi kontradiksi dengan $K \in \mathfrak{R}$, sehingga pengandaian harus diingkar. Jadi, $\Phi$ tidak memenuhi kondisi- $\Delta_{2} \cdot \square$ 
Selanjutnya, untuk sebarang fungsi Orlicz $\Phi$ yang memenuhi kondisi- $\Delta_{2}$, didefinisikan fungsi $\Psi: \mathfrak{R} \rightarrow \mathfrak{R}^{+}$ dengan

$$
\Psi(y)=\sup \{x|y|-\Phi(x): x>0\},
$$

untuk setiap $y \in \mathfrak{R}$. Fungsi $\Psi$ tersebut juga merupakan fungsi Orlicz. Fungsi $\Psi$ ini selanjutnya disebut sebagai komplemen fungsi $\Phi$. Jadi, jika $\Psi$ komplemen fungsi $\Phi$, maka

$$
\Psi(y)=\sup \{x|y|-\Phi(x): x>0\} .
$$

Akibatnya, untuk setiap $x>0$, berlaku $\Psi(y) \geq x|y|-\Phi(x)$, untuk setiap $y \in \mathfrak{R}$ $\Leftrightarrow x|y| \leq \Psi(y)+\Phi(x), \quad$ untuk setiap $y \in \mathfrak{R}$.

Karena $\quad \Phi(|x|)=\Phi(x)$, maka untuk setiap $x, y \in \mathfrak{R}$ berlaku pula

$$
|x y| \leq \Phi(x)+\Psi(y) .
$$

Selanjutnya, ketaksamaan ini disebut sebagai Ketaksamaan Young.

Diberikan $\Psi$ komplemen fungsi Orlicz $\Phi$. Fungsi $\Psi: \mathfrak{R} \rightarrow \mathfrak{R}^{+}$dikatakan konveks, jika untuk setiap $y, z \in \mathfrak{R}$ dan $\alpha, \beta \in[0,1]$ dengan $\alpha+\beta=1$ berlaku

$$
\Psi(\alpha y+\beta z) \leq \alpha \Psi(y)+\beta \Psi(z) .
$$

Kekonveksan fungsi $\Psi$, komplemen fungsi Orlicz $\Phi$, dijamin dalam teorema berikut.

Teorema 1. Jika $\Psi$ komplemen fungsi Orlicz $\Phi$, maka $\Psi$ konveks.
Bukti. Diambil sebarang $y, z \in \mathfrak{R}$ dan $\alpha, \beta \in[0,1]$ dengan $\alpha+\beta=1$. Karena $\Psi$ komplemen $\Phi$, maka

$$
\begin{gathered}
\Psi(y)=\sup \{x|y|-\Phi(x): x>0\} \text { dan } \\
\Psi(z)=\sup \{x|z|-\Phi(x): x>0\} .
\end{gathered}
$$

Berarti untuk setiap $x>0$, berlaku

$$
\begin{gathered}
\Psi(y) \geq x|y|-\Phi(x) \text { dan } \\
\Psi(z) \geq x|z|-\Phi(x) .
\end{gathered}
$$

Akibatnya, untuk setiap $x>0$ dan $\alpha, \beta \in[0,1]$ dengan $\alpha+\beta=1$ diperoleh

$$
\begin{aligned}
\alpha \Psi & (y)+\beta \Psi(z) \geq \alpha(x|y|-\Phi(x))+\beta(x|z|-\Phi(x)) \\
& =\alpha x|y|-\alpha \Phi(x)+\beta x|z|-\beta \Phi(x) \\
& =\alpha x|y|+\beta x|z|-\alpha \Phi(x)-\beta \Phi(x) \\
& =x(\alpha|y|+\beta|z|)-(\alpha+\beta) \Phi(x) \\
& =x(|\alpha y|+|\beta z|)-\Phi(x) \\
& \geq x|\alpha y+\beta z|-\Phi(x) .
\end{aligned}
$$

Karena

$\Psi(\alpha y+\beta z)=\sup \{x|\alpha y+\beta z|-\Phi(x): x>0\}$, maka $\Psi(\alpha y+\beta z) \leq \alpha \Psi(y)+\beta \Psi(z)$.

Dengan kata lain, terbukti $\Psi$ konveks.

Diberikan fungsi Orlicz $\Phi$ yang memenuhi kondisi- $\Delta_{2}$ dan ruang linear $X_{\Phi} \subset \omega$ merupakan ruang barisan yang dibangkitkan oleh fungsi Orlicz $\Phi$. Selanjutnya, ruang linear $X_{\Phi} \subset \omega$ cukup disebut ruang $X_{\Phi}$. Didefinisikan fungsi $\|\cdot\|_{(\Phi)}: X_{\Phi} \rightarrow \mathfrak{R}$ dengan 


$$
\|x\|_{(\Phi)}=\inf \left\{\varepsilon>0: \sum_{n=1}^{\infty} \Phi\left(\frac{x_{n}}{\varepsilon}\right) \leq 1\right\},
$$

untuk setiap $x \in X_{\Phi}$. Apabila $\Phi$ konveks, maka \|\|$_{(\Phi)}$ merupakan norma pada $X_{\Phi}$.

Norma \|\|$_{(\Phi)}$ pada $X_{\Phi}$ selanjutnya disebut norma Luxemburg (Luxemburg norm).

Selanjutnya akan dibahas tentang pengertian konvergen (biasa), barisan Cauchy, $\Phi$-konvergen, dan $\Phi$-Cauchy pada $X_{\Phi}$. Fungs Orlicz $\Phi$ yang dimaksud di dalam pembahasan ini adalah fungsi Orlicz yang memenuhi kondisi- $\Delta_{2}$.

Definisi 3. Diberikan ruang $X_{\Phi}$ dengan $\Phi$ fungsi Orlicz. Barisan $\left(x^{(t)}\right) \subseteq X_{\Phi}$ dikatakan konvergen (biasa) ke $x \in X_{\Phi}$, ditulis $x^{(t)} \longrightarrow x$, jika untuk setiap $\varepsilon>0$, terdapat $t_{0} \in \mathrm{N}$ sehingga untuk setiap $t \in \mathrm{N}$ dengan $t \geq t_{0} \quad$ berlaku $\left\|x^{(t)}-x\right\|_{(\Phi)}<\varepsilon$.

Definisi 4. Diberikan ruang $X_{\Phi}$ dengan $\Phi$ fungsi Orlicz. Barisan $\left(x^{(t)}\right) \subseteq X_{\Phi}$ disebut barisan Cauchy jika untuk setiap $\varepsilon>0$, terdapat $t_{0} \in \mathrm{N}$ sehingga untuk setiap $t, p \in \mathrm{N}$ dengan $t, p \geq t_{0}$ berlaku $\left\|x^{(t)}-x^{(p)}\right\|_{(\Phi)}<\varepsilon$.
Definisi 5. Diberikan ruang $X_{\Phi}$ dengan $\Phi$ fungsi Orlicz. Barisan $\left(x^{(t)}\right) \subseteq X_{\Phi}$ dikatakan $\Phi$-konvergen $\quad \Phi$ convergence) ke $x \in X_{\Phi}$, ditulis $x^{(t)} \stackrel{\Phi}{\longrightarrow} x$, jika untuk setiap $\varepsilon>0$, terdapat $t_{0} \in \mathrm{N}$ sehingga untuk setiap $t \in \square \quad$ dengan $t \geq t_{0} \quad$ berlaku $\sum_{n=1}^{\infty} \Phi\left(x_{n}^{(t)}-x_{n}\right)<\varepsilon$.

Dengan kata lain, $\lim _{t \rightarrow \infty} \sum_{n=1}^{\infty} \Phi\left(x_{n}^{(t)}-x_{n}\right)=0$.

Dalam hal ini, x disebut limit- $\Phi$ barisan $\left(x^{(t)}\right)$.

Definisi 6. Diberikan ruang $X_{\Phi}$ dengan $\Phi$ fungsi Orlicz. Barisan $\left(x^{(t)}\right) \subseteq X_{\Phi}$ disebut barisan $\Phi$-Cauchy ( $\Phi$-Cauchy sequence) jika untuk setiap $\varepsilon>0$, terdapat $t_{0} \in \mathrm{N}$ sehingga untuk setiap $t, p \in \mathrm{N} \quad$ dengan $\quad t, p \geq t_{0} \quad$ berlaku $\sum_{n=1}^{\infty} \Phi\left(x_{n}^{(t)}-x_{n}^{(p)}\right)<\varepsilon$.

Selanjutnya, ketunggalan limit- $\Phi$ barisan $\left(x^{(t)}\right)$, dijamin dalam teorema berikut.

Teorema 2. Diberikan fungsi Orlicz $\Phi$ . Jika limit- $\Phi$ barisan $\left(x^{(t)}\right)$ ada, maka nilai limit- $\Phi$ nya tunggal. 
Bukti. Dimisalkan $x, y \in X_{\Phi}$ merupakan limit- $\Phi$ barisan $\left(x^{(t)}\right) \subseteq X_{\Phi} . \quad$ Karena $\Phi \in \Delta_{2}$, maka terdapat $M>0$ sehingga $\Phi(2 x) \leq M \Phi(x)$, untuk setiap $x \geq 0$.

Diambil sebarang $\varepsilon>0$, maka $\frac{\varepsilon}{M}>0$. Karena barisan $\left(x^{(t)}\right) \Phi$-konvergen ke $\mathrm{x}$ dan y, maka terdapat $t_{1}, t_{2} \in \square$ sehingga $\sum_{n=1}^{\infty} \Phi\left(x_{n}^{(t)}-x_{n}\right)<\frac{\varepsilon}{M}$, untuk setiap $t \geq t_{1}$ dan $\sum_{n=1}^{\infty} \Phi\left(x_{n}^{(t)}-y_{n}\right)<\frac{\varepsilon}{M}$, untuk setiap $t \geq t_{2}$.

Diambil $t_{0}=\max \left\{t_{1}, t_{2}\right\}$, maka untuk sebarang $t \geq t_{0}$ berlaku

$$
\begin{aligned}
\sum_{n=1}^{\infty} & \left(x_{n}-y_{n}\right)=\sum_{n=1}^{\infty} \Phi\left(2 \cdot \frac{1}{2}\left(x_{n}-x_{n}^{(t)}+x_{n}^{(t)}-y_{n}\right)\right) \\
& \leq \sum_{n=1}^{\infty} M \Phi\left(\frac{1}{2}\left(x_{n}-x_{n}^{(t)}\right)+\frac{1}{2}\left(x_{n}^{(t)}-y_{n}\right)\right) \\
& \leq \sum_{n=1}^{\infty} M\left(\frac{1}{2} \Phi\left(x_{n}-x_{n}^{(t)}\right)+\frac{1}{2} \Phi\left(x_{n}^{(t)}-y_{n}\right)\right) \\
& =\frac{M}{2}\left(\sum_{n=1}^{\infty} \Phi\left(x_{n}-x_{n}^{(t)}\right)+\sum_{n=1}^{\infty} \Phi\left(x_{n}^{(t)}-y_{n}\right)\right) \\
& <\frac{M}{2}\left(\frac{\varepsilon}{M}+\frac{\varepsilon}{M}\right)=\varepsilon .
\end{aligned}
$$

Karena berlaku untuk sebarang $\varepsilon>0$, maka $\sum_{n=1}^{\infty} \Phi\left(x_{n}-y_{n}\right)=0$.

Akibatnya, $x_{n}-y_{n}=0$, untuk setiap $n \in$ N. Dengan kata lain, $x=y$.

Hubungan $\Phi$-konvergen dengan barisan $\Phi$-Cauchy akan dijelaskan dalam teorema berikut.
Teorema 3. Diberikan ruang $X_{\Phi}$ dengan $\Phi$ fungsi Orlicz dan konveks. Jika $\left(x^{(t)}\right) \subseteq X_{\Phi}$ barisan $\Phi$-konvergen, maka $\left(x^{(t)}\right)$ barisan $\Phi$-Cauchy.

Bukti. Karena $\Phi \in \Delta_{2}$, maka tedapat $M>0$ sehingga $\Phi(2 x) \leq M \Phi(x)$, untuk setiap $x \geq 0$. Diambil sebarang $\varepsilon>0$, maka $\frac{\varepsilon}{M}>0$. Karena barisan $\left(x^{(t)}\right) \Phi-$ konvergen, katakan ke $x \in X_{\Phi}$, maka terdapat $t_{0} \in \mathrm{N}$ sehingga untuk setiap $t \in$ $\mathrm{N}$ dengan $t \geq t_{0} \quad$ berlaku $\sum_{n=1}^{\infty} \Phi\left(x_{n}^{(t)}-x_{n}\right)<\frac{\varepsilon}{M}$. Jadi untuk setiap $t, p \geq t_{0}$ berlaku

$$
\begin{aligned}
& \sum_{n=1}^{\infty} \Phi\left(x_{n}^{(t)}-x_{n}^{(p)}\right)=\sum_{n=1}^{\infty} \Phi\left(2 \cdot \frac{1}{2}\left(x_{n}^{(t)}-x_{n}+x_{n}-x_{n}^{(p)}\right)\right) \\
& \leq \sum_{n=1}^{\infty} M \Phi\left(\frac{1}{2}\left(x_{n}^{(t)}-x_{n}\right)+\frac{1}{2}\left(x_{n}-x_{n}^{(p)}\right)\right) \\
& \leq M \sum_{n=1}^{\infty}\left(\frac{1}{2} \Phi\left(x_{n}^{(t)}-x_{n}\right)+\frac{1}{2} \Phi\left(x_{n}-x_{n}^{(p)}\right)\right) \\
& =\frac{M}{2}\left(\sum_{n=1}^{\infty} \Phi\left(x_{n}^{(t)}-x_{n}\right)+\sum_{n=1}^{\infty} \Phi\left(x_{n}-x_{n}^{(p)}\right)\right) \\
& \quad<\frac{M}{2}\left(\frac{\varepsilon}{M}+\frac{\varepsilon}{M}\right)=\varepsilon .
\end{aligned}
$$

Dengan kata lain, $\left(x^{(t)}\right)$ barisan $\Phi$ Cauchy.

Konvers Teorema 3 belum tentu benar dan berikut ini diberikan contoh penyangkalnya.

Contoh 5. Diberikan himpunan $X=\{(a, 0,0, \ldots): a \in \mathfrak{R}\} \quad$ dan himpunan 
$X_{\mathbf{Q}}=\{(a, 0,0, \ldots): a \in \mathbf{Q}\}$. Himpunan $X_{\mathbf{Q}}$ merupakan ruang bagian dari $\mathrm{X}$.

Didefinisikan fungsi $\Phi: \Re \rightarrow \mathfrak{R}^{+}$ dengan $\Phi(t)=|t|$, untuk setiap $t \in \Re$. Fungsi $\Phi$ merupakan fungsi Orlicz sebab $\Phi$ kontinu pada $\Re, \Phi$ fungsi genap, $\Phi$ naik pada $\mathfrak{R}^{+}$, dan $\Phi(t)=0 \Leftrightarrow t=0$. Fungsi $\Phi$ juga memenuhi kondisi- $\Delta_{2}$.

Diberikan barisan $\left(x^{(k)}\right) \subseteq X_{\mathbf{Q}}$ dengan $x_{n}^{(k)}=\left(1+\frac{1}{n}\right)^{n}, \quad$ untuk $\quad$ setiap $\quad n \in \mathrm{N}$.

Akan ditunjukkan $\left(x^{(k)}\right)$ merupakan barisan $\Phi$-Cauchy di dalam $X_{\mathbf{Q}}$. Diambil sebarang $m, n \in \mathrm{N}$. Diasumsikan $m>n$.

$$
\begin{aligned}
& \sum_{k=1}^{\infty} \Phi\left(x_{m}^{(k)}-x_{n}^{(k)}\right)=\Phi\left(x_{m}^{(1)}-x_{n}^{(1)}\right) \\
& =\left|x_{m}^{(1)}-x_{n}^{(1)}\right|=\left|\left(1+\frac{1}{m}\right)^{m}-\left(1+\frac{1}{n}\right)^{n}\right| \\
& =\left|\sum_{k=0}^{m} \mathrm{C}_{k}^{m} 1^{m-k}\left(\frac{1}{m}\right)^{k}-\sum_{j=0}^{n} \mathrm{C}_{j}^{n} 1^{n-j}\left(\frac{1}{n}\right)^{j}\right| \\
& =\mid\left(1+\frac{1}{1 !}+\frac{1}{2 !}\left(1-\frac{1}{m}\right)+\frac{1}{3 !}\left(1-\frac{1}{m}\right)\left(1-\frac{2}{m}\right)+\ldots\right. \\
& \left.\quad+\frac{1}{n !}\left(1-\frac{1}{m}\right)\left(1-\frac{2}{m}\right) \ldots\left(1-\frac{n-1}{m}\right)+\ldots+\left(\frac{1}{m}\right)^{m}\right)
\end{aligned}
$$

$-\left(1+\frac{1}{1 !}+\frac{1}{2 !}\left(1-\frac{1}{n}\right)+\frac{1}{3 !}\left(1-\frac{1}{n}\right)\left(1-\frac{2}{n}\right)+\ldots+\frac{1}{n !} \frac{n !}{n^{n}}\right)$ nnacam $\begin{array}{rrr}\text { fungsi } & \text { Orlicz } & \Phi \text { dengan berbagai } \\ \text { nelanjutnya, akan }\end{array}$ $\leq\left|\frac{1}{2 !}\left(\frac{1}{n}-\frac{1}{m}\right)+\frac{1}{3 !}\left(\frac{1}{n}-\frac{1}{m}\right)+\ldots+\frac{1}{n !}\left(\frac{1}{n}-\frac{1}{m}\right)\right|$ $<\left|\frac{1}{2 !}+\frac{1}{3 !}+\ldots+\frac{1}{n !}\right|\left|\frac{1}{n}\right| \rightarrow 0$, untuk $n \rightarrow \infty$.
Karena $\lim _{n \rightarrow \infty}\left(\frac{1}{2 !}+\frac{1}{3 !}+\ldots+\frac{1}{n !}\right)=a$, untuk suatu $a \in \mathfrak{R}$, maka untuk $n, m \rightarrow \infty$ berlaku $\Phi\left(x_{m}-x_{n}\right) \rightarrow 0$. Dengan kata lain, $\left(x^{(k)}\right)$ barisan Cauchy- $\Phi$ di $X_{\mathbf{Q}}$.

Di lain pihak,

$\lim _{n \rightarrow \infty} x_{n}=\lim _{n \rightarrow \infty}\left(1+\frac{1}{n}\right)^{n}=e$,

dengan e bilangan alam. Oleh karena itu,

$\lim _{n \rightarrow \infty}\left|x_{n}-e\right|=0 \Leftrightarrow \lim _{n \rightarrow \infty} \Phi\left(x_{n}-e\right)=0$.

Karena $e \notin X_{\mathbf{Q}}$, maka barisan $\left(x^{(k)}\right)$ tidak $\Phi$-konvergen di dalam $X_{\mathbf{Q}}$.

Berdasarkan Contoh 5 dapat disimpulkan bahwa setiap barisan $\Phi$ Cauchy belum tentu konvergen- $\Phi$. Selanjutnya, ruang $X_{\Phi}$ yang setiap barisan $\Phi$-Cauchy nya bersifat $\Phi$ konvergen disebut ruang $\Phi$-lengkap, seperti diberikan dalam definisi berikut.

Definisi 7. Diberikan fungsi Orlicz $\Phi$. Ruang $X_{\Phi}$ dikatakan $\Phi$-lengkap jika setiap barisan $\Phi$-Cauchy di dalamnya $\Phi$-konvergen.

Ruang $X_{\Phi}$ dibangkitkan oleh diberikan contoh dari ruang $X_{\Phi}$, yaitu ruang $\tilde{\ell}_{\Phi}$ dan $\ell_{\Phi}$. Pendefinisian ruang $\tilde{\ell}_{\Phi}$ mengacu pada ruang barisan yang 
dikonstruksi oleh Lindenstrauss dan Tzafriri, yaitu:

$$
\ell(\Phi)=\left\{\begin{array}{r}
x=\left(x_{k}\right) \in \omega: \sum_{k=1}^{\infty} \Phi\left(\frac{\left|x_{k}\right|}{\varepsilon}\right)<\infty, \\
\text { untuk suatu } \varepsilon>0
\end{array}\right\}
$$

dengan $\omega$ koleksi semua barisan bilangan real dan $\Phi$ fungsi Orlicz yang bersifat konveks. Diberikan fungsi Orlicz $\Phi$, didefinisikan himpunan

$$
\tilde{\ell}_{\Phi}=\left\{x=\left(x_{n}\right): \sum_{n=1}^{\infty} \Phi\left(x_{n}\right)<\infty\right\} .
$$

Himpunan $\quad \tilde{\ell}_{\Phi}$ ini disebut Kelas Barisan Orlicz (Orlicz Sequence Class). Himpunan $\tilde{\ell}_{\Phi}$ merupakan ruang linear. Apabila $\Phi$ konveks, maka ruang $\tilde{\ell}_{\Phi}$ merupakan ruang bernorma terhadap norma Luxemburg \|\|$_{(\Phi)}$ dengan definisi

$$
\|x\|_{(\Phi)}=\inf \left\{\varepsilon>0: \sum_{n=1}^{\infty} \Phi\left(\frac{x_{n}}{\varepsilon}\right) \leq 1\right\},
$$

untuk setiap $x \in \tilde{\ell}_{\Phi}$.

Diberikan fungsi Orlicz $\Phi$ dan $\Psi$ komplemen fungsi $\Phi$, didefinisikan himpunan

$$
\ell_{\Phi}=\left\{x=\left(x_{n}\right): \sum_{n=1}^{\infty}\left|x_{n} y_{n}\right|<\infty, \forall y \in \tilde{\ell}_{\Psi}\right\}
$$

Himpunan $\ell_{\Phi}$ tersebut juga merupakan ruang linear.

Diberikan fungsi Orlicz $\Phi$ dan $\Psi$ komplemen fungsi $\Phi$. Didefinisikan fungsi $\|\cdot\|_{\Phi}: \ell_{\Phi} \rightarrow \Re$ dengan

$$
\|x\|_{\Phi}=\sup \left\{\sum_{n=1}^{\infty}\left|x_{n} y_{n}\right|: y \in \tilde{\ell}_{\Psi}, \sum_{n=1}^{\infty} \Psi\left(y_{n}\right) \leq 1\right\}
$$

untuk setiap $x \in \ell_{\Phi}$.

Fungsi $\|.\|_{\Phi}$ merupakan norma pada $\ell_{\Phi}$ yang selanjutnya disebut norma Orlicz (Orlicz norm). Ruang $\quad \ell_{\Phi} \quad$ yang dilengkapi dengan norma Orlicz \|\|$_{\Phi}$, ditulis $\left(\ell_{\Phi},\|\|_{\Phi}\right)$, disebut ruang barisan Orlicz (Orlicz Sequence Space).

Jika $\Phi \in \Delta_{2}$, maka $\tilde{\ell}_{\Phi}=\ell_{\Phi}$ dan norma Luxemburg \|\|$_{(\Phi)}$ ekuivalen dengan norma Orlicz $\|\cdot\|_{\Phi}$ pada $\ell_{\Phi}$.

Di dalam ruang barisan Orlicz juga berlaku Ketaksamaan Holder, yaitu:

$$
\sum_{n=1}^{\infty}\left|x_{n} y_{n}\right| \leq\|x\|_{\Phi}\|y\|_{\Psi},
$$

untuk setiap $x \in \ell_{\Phi}$ dan $y \in \ell_{\Psi}$.

Selanjutnya, pembahasan pada paper ini akan lebih ditekankan pada ruang $\ell_{\Phi}$. Hubungan ekuivalensi antara konvergen dan $\Phi$-konvergen dalam ruang barisan Orlicz $\left(\ell_{\Phi},\|\|_{\Phi}\right)$ akan dijelaskan dalam teorema berikut. Sebagai pendukung bukti teorema tersebut, terlebih dahulu diberikan teorema Kesamaan Young dan lemma sebagai berikut.

Teorema 4. Jika $\Phi$ dan $\Psi$ fungsi Orlicz, maka $|x y|=\Phi(x)+\Psi(y)$, untuk setiap $x, y \in \mathfrak{R}$. 
Bukti. Fungsi $\Phi: \mathfrak{R} \rightarrow \mathfrak{R}^{+}$dengan definisi $\Phi(x)=\int_{0}^{|x|} f() t \quad$ merupakan fungsi Orlicz dengan fungsi $f: \mathfrak{R}^{+} \rightarrow \mathfrak{R}^{+}$ bersifat $f(x)=0 \Leftrightarrow x=0$, f kontinu dan naik pada $\mathfrak{R}^{+}$. Fungsi $\Psi: \mathfrak{R} \rightarrow \mathfrak{R}^{+}$dengan definisi $\quad \Psi(x)=\int_{0}^{|x|}(g) \quad$ juga merupakan fungsi Orlicz dengan $g=f^{-1}$. Diambil $y=f(x)$, untuk setiap $x \in \mathfrak{R}$, maka berlaku

$$
\begin{aligned}
\Phi(x)+\Psi(y) & =\int_{0}^{|x|} f(t) d t+\int_{0}^{|y|} g(s) d s \\
& =\int_{0}^{|x|} f(t) d t+\int_{0}^{|f(x)|} g(s) d s \\
& =|x f(x)|=|x y| .
\end{aligned}
$$

Lemma 5. Diberikan fungsi Orlicz $\Phi$ dan $\Psi$ komplemen fungsi $\Phi$. Jika $x \in \ell_{\Phi}$ dengan $\|x\|_{\Phi} \leq 1$, maka $x \in \tilde{\ell}_{\Phi}$ $\operatorname{dan} \sum_{n=1}^{\infty} \Phi\left(x_{n}\right) \leq\|x\|_{\Phi}$.

Bukti. Diambil sebarang $x \in \ell_{\Phi}$ dengan $\|x\|_{\Phi} \leq 1$. Mengacu pada Teorema 4, maka untuk setiap $y \in \tilde{\ell}_{\Psi}$ dengan $\sum_{n=1}^{\infty} \Psi\left(y_{n}\right) \leq 1$ berlaku

$$
\begin{aligned}
\sum_{n=1}^{\infty} \Phi\left(x_{n}\right) & \leq \sum_{n=1}^{\infty} \Phi\left(x_{n}\right)+\sum_{n=1}^{\infty} \Psi\left(y_{n}\right) \\
& =\sum_{n=1}^{\infty}\left|x_{n} y_{n}\right| \leq\|x\|_{\Phi} .
\end{aligned}
$$

Teorema 6. Diberikan ruang barisan Orlicz $\left(\ell_{\Phi},\|\cdot\|_{\Phi}\right)$ dengan $\Phi$ fungsi Orlicz dan $\Psi$ komplemen fungsi $\Phi$ yang memenuhi Kesamaan Young. Kedua pernyataan berikut ini ekuivalen. Barisan $\left(x^{(t)}\right) \subseteq \ell_{\Phi}$ konvergen ke $x \in \ell_{\Phi}$. Barisan $\left(x^{(t)}\right) \subseteq \ell_{\Phi} \quad \Phi$-konvergen ke $x \in \ell_{\Phi}$.

\section{Bukti.}

$(i \Rightarrow i i)$

Diambil sebarang $\varepsilon>0$, dengan asumsi $\varepsilon \in(0,1)$. Karena diketahui $\left(x^{(t)}\right) \subseteq \ell_{\Phi}$ konvergen ke $x \in \ell_{\Phi}$, maka terdapat $T \in \square \quad$ sehingga untuk setiap $t \in \square$ dengan $t \geq T$ berlaku $\left\|x^{(t)}-x\right\|_{\Phi}<\varepsilon$. Karena $\ell_{\Phi}$ ruang linear, maka $x^{(t)}-x \in \ell_{\Phi}, \quad$ untuk setiap $t=1,2, \ldots$ Akibatnya, menurut Lemma 5, $\sum_{n=1}^{\infty} \Phi\left(x_{n}^{(t)}-x_{n}\right) \leq\left\|x^{(t)}-x\right\|_{\Phi}<\varepsilon, \quad$ untuk setiap $t \geq T$. Dengan kata lain, $\left(x^{(t)}\right) \Phi-$ konvergen ke $x \in \ell_{\Phi}$. $(i i \Rightarrow i)$ 
Diambil sebarang $\varepsilon>0$, maka menurut sifat Archimedes terdapat $k \in \square$ dengan $k \neq 1$ sehingga $\frac{1}{2^{k-1}}<\varepsilon$. Diketahui $\left(x^{(t)}\right)$ $\Phi$-konvergen ke $x \in \ell_{\Phi}, \quad$ berarti $\sum_{n=1}^{\infty} \Phi\left(x_{n}^{(t)}-x_{n}\right) \rightarrow 0, \quad$ untuk $\quad t \rightarrow \infty$. Dengan kata lain, $\lim _{t \rightarrow \infty} \sum_{n=1}^{\infty} \Phi\left(x_{n}^{(t)}-x_{n}\right)=0$. Karena $\Phi \in \Delta_{2}$, maka terdapat $M>0$ sehingga $\lim _{t \rightarrow \infty} \sum_{n=1}^{\infty} \Phi\left(2^{k}\left(x_{n}^{(t)}-x_{n}\right)\right) \leq \lim _{t \rightarrow \infty} \sum_{n=1}^{\infty} M^{k} \Phi\left(x_{n}^{(t)}-x_{n}\right)$ $=M^{k} \lim _{t \rightarrow \infty} \sum_{n=1}^{\infty} \Phi\left(x_{n}^{(t)}-x_{n}\right)=M^{k} .0=0$.

Berarti $\lim _{t \rightarrow \infty} \sum_{n=1}^{\infty} \Phi\left(2^{k}\left(x_{n}^{(t)}-x_{n}\right)\right)=0 . \quad$ Jika diambil $\varepsilon=1>0$, maka terdapat $T \in \mathrm{N}$ sehingga untuk setiap $t \in \mathrm{N}$ dengan $t \geq T \quad$ berlaku $\quad \sum_{n=1}^{\infty} \Phi\left(2^{k}\left(x_{n}^{(t)}-x_{n}\right)\right)<1$. Jadi untuk setiap $t \geq T$ diperoleh $\left\|2^{k}\left(x^{(t)}-x\right)\right\|_{\Phi}$
$=\sup \left\{\sum_{n=1}^{\infty}\left|2^{k}\left(x_{n}^{(t)}-x_{n}\right) y_{n}\right|: y \in \tilde{\ell}_{\Psi}, \sum_{n=1}^{\infty} \Psi\left(y_{n}\right) \leq 1\right\}$
$=\sup \left\{\sum_{n=1}^{\infty}\left|2^{k}\left(x_{n}^{(t)}-x_{n}\right)\right|\left|y_{n}\right|: y \in \tilde{\ell}_{\Psi}, \sum_{n=1}^{\infty} \Psi\left(y_{n}\right) \leq 1\right\}$
$\leq \sup \left\{\sum_{n=1}^{\infty} \Phi\left(2^{k}\left(x_{n}^{(t)}-x_{n}\right)\right)+\sum_{n=1}^{\infty} \Psi\left(y_{n}\right):\right.$
$\left.\quad y \in \tilde{\ell}_{\Psi}, \sum_{n=1}^{\infty} \Psi\left(y_{n}\right) \leq 1\right\}$
$\leq \sup \left\{\sum_{n=1}^{\infty} \Phi\left(2^{k}\left(x_{n}^{(t)}-x_{n}\right)\right): y \in \tilde{\ell}_{\Psi}, \sum_{n=1}^{\infty} \Psi\left(y_{n}\right) \leq 1\right\}$ $+\sup \left\{\sum_{n=1}^{\infty} \Psi\left(y_{n}\right): y \in \tilde{\ell}_{\Psi}, \sum_{n=1}^{\infty} \Psi\left(y_{n}\right) \leq 1\right\}$ $=1+1=2$.

Akibatnya,

$$
\left|2^{k}\right|\left\|\left(x^{(t)}-x\right)\right\|_{\Phi}=\left\|2^{k}\left(x^{(t)}-x\right)\right\|_{\Phi} \leq 2,
$$

untuk setiap $\varepsilon>0$.

Dengan kata lain, $\left(x^{(t)}\right)$ konvergen ke $x \in \ell_{\Phi} \cdot \square$

Selanjutnya akan diberikan teorema yang menjelaskan hubungan ekuivalensi antara barisan Cauchy dan barisan $\Phi$ Cauchy dalam ruang barisan Orlicz $\left(\ell_{\Phi},\|\cdot\|_{\Phi}\right)$.

Teorema 7. Diberikan ruang barisan Orlicz $\left(\ell_{\Phi},\|\cdot\|_{\Phi}\right)$ dengan $\Phi$ fungsi Orlicz dan $\Psi$ komplemen fungsi $\Phi$ yang memenuhi Kesamaan Young. Kedua pernyataan berikut ini ekuivalen.

i. Barisan $\left(x^{(t)}\right) \subseteq \ell_{\Phi}$ barisan Cauchy.

ii. $\operatorname{Barisan}\left(x^{(t)}\right) \subseteq \ell_{\Phi}$ barisan $\Phi$ Cauchy.

\section{Bukti.}

$(i \Rightarrow i i)$ Diambil sebarang barisan Cauchy $\left(x^{(t)}\right)$ di dalam $\left(\ell_{\Phi},\|\|_{\Phi}\right)$, berarti untuk setiap $\varepsilon>0$, dengan asumsi $\varepsilon \in(0,1)$, terdapat $t_{0} \in \mathrm{N}$ sehingga untuk setiap $t, p \in \mathrm{N}$ dengan $t, p \geq t_{0}$ berlaku $\left\|x^{(t)}-x^{(p)}\right\|_{\Phi}<\varepsilon . \quad$ Karena $\quad \ell_{\Phi} \quad$ ruang 
linear, maka $x^{(t)}-x^{(p)} \in \ell_{\Phi}$, untuk setiap $t=1,2, \ldots$ dan $p=1,2, \ldots$

Akibatnya, menurut Lemma 5

$$
\sum_{n=1}^{\infty} \Phi\left(x_{n}^{(t)}-x_{n}^{(p)}\right) \leq\left\|x^{(t)}-x^{(p)}\right\|_{\Phi}<\varepsilon .
$$

Jadi, terbukti $\left(x^{(t)}\right)$ barisan $\Phi$-Cauchy.

$(i i \Rightarrow i)$ Diambil sebarang $\varepsilon>0$, maka

terdapat $k \in \mathrm{N}$ dan $k \neq 1$ sehingga

$\frac{1}{2^{k-1}}<\varepsilon . \quad$ Karena $\Phi \in \Delta_{2} \quad$ dan $\quad\left(x^{(t)}\right)$

barisan $\Phi$-Cauchy, maka

$$
\begin{aligned}
\lim _{t \rightarrow \infty} \sum_{n=1}^{\infty} \Phi\left(2^{k}\left(x_{n}^{(t)}-x_{n}^{(p)}\right)\right) & \leq \lim _{t \rightarrow \infty} \sum_{n=1}^{\infty} M \Phi\left(x_{n}^{(t)}-x_{n}^{(p)}\right) \\
& =M \lim _{t \rightarrow \infty} \sum_{n=1}^{\infty} \Phi\left(x_{n}^{(t)}-x_{n}^{(p)}\right) \\
& =M .0=0 .
\end{aligned}
$$

Berarti jika diambil $\varepsilon_{0}=1>0$, maka terdapat $t_{0} \in \mathrm{N}$ sehingga untuk setiap $t, p \in \mathrm{N} \quad$ dengan $\quad t, p \geq t_{0} \quad$ berlaku $\lim _{t \rightarrow \infty} \sum_{n=1}^{\infty} \Phi\left(2^{k}\left(x_{n}^{(t)}-x_{n}^{(p)}\right)\right)<1$.

Jadi, untuk setiap $t, p \geq t_{0}$ berlaku

$$
\begin{aligned}
& \left\|2^{k}\left(x^{(t)}-x^{(p)}\right)\right\|_{\Phi} \\
& =\sup \left\{\sum_{n=1}^{\infty}\left|2^{k}\left(x_{n}^{(t)}-x_{n}^{(p)}\right) \cdot y_{n}\right|: y \in \tilde{\ell}_{\Psi}, \sum_{n=1}^{\infty} \Psi\left(y_{n}\right) \leq 1\right\} \\
& =\sup \left\{\sum_{n=1}^{\infty}\left|2^{k}\left(x_{n}^{(t)}-x_{n}^{(p)}\right)\right|\left|y_{n}\right|: y \in \tilde{\ell}_{\Psi}, \sum_{n=1}^{\infty} \Psi\left(y_{n}\right) \leq 1\right\} \\
& \leq \sup \left\{\sum_{n=1}^{\infty} \Phi\left(2^{k}\left(x_{n}^{(t)}-x_{n}^{(p)}\right)\right)+\sum_{n=1}^{\infty} \Psi\left(x_{n}\right)\right. \\
& \left.\quad: y \in \tilde{\ell}_{\Psi}, \sum_{n=1}^{\infty} \Psi\left(y_{n}\right) \leq 1\right\} \\
& <1+1=2 .
\end{aligned}
$$

Akibatnya,

$$
\begin{aligned}
& \left|2^{k}\right|\left\|x^{(t)}-x^{(p)}\right\|_{\Phi}=\left\|2^{k}\left(x^{(t)}-x^{(p)}\right)\right\|_{\Phi}<2 \\
& \Leftrightarrow\left\|x^{(t)}-x^{(p)}\right\|_{\Phi}<\frac{2}{2^{k}}=\frac{1}{2^{k-1}}<\varepsilon .
\end{aligned}
$$

Jadi, terbukti $\left(x^{(t)}\right)$ barisan Cauchy.

Ruang barisan Orlicz $\left(\ell_{\Phi},\|\|_{\Phi}\right)$ merupakan ruang bernorma yang lengkap atau ruang Banach seperti ditunjukkan dalam teorema berikut.

Teorema 8. Diberikan fungsi Orlicz $\Phi$ dan $\Psi$ komplemen fungsi $\Phi$. Ruang barisan Orlicz $\left(\ell_{\Phi},\|\|_{\Phi}\right)$ merupakan ruang Banach.

Bukti. Diambil sebarang $\left(x^{(t)}\right)$ barisan Cauchy di dalam $\left(\ell_{\Phi},\|\|_{\Phi}\right)$, berarti untuk setiap $\varepsilon>0$, terdapat $t_{0} \in \square$ sehingga untuk setiap $y \in \tilde{\ell}_{\Psi}$ dengan $\sum_{n=1}^{\infty} \Psi\left(y_{n}\right) \leq 1$ dan untuk setiap $t, p \geq t_{0}$ berlaku

$$
\sum_{n=1}^{\infty}\left|x_{n}^{(t)}-x_{n}^{(p)}\right|\left|y_{n}\right|<\varepsilon
$$

Selanjutnya, untuk setiap $n \in \mathrm{N}$ dibentuk $A_{n}=\{n\}$. Jadi, $\mathrm{N}=\bigcup_{n=1}^{\infty} A_{n}$. Dari sifat-sifat $\Psi$, dapat dipilih bilangan $k>0$ sehingga $0<\Psi(k) \leq 1$.

Untuk setiap $n \in \mathrm{N}$ dan untuk setiap $m \in$ $\mathrm{N}$ dapat didefinisikan

$$
y_{n}^{(m)}=\left\{\begin{array}{l}
k, n \in A_{m} \\
0, n \notin A_{m}
\end{array} .\right.
$$


Diperoleh $\left(y_{n}^{(m)}\right) \in \tilde{\ell}_{\Psi}, \quad$ untuk setiap $m \geq 1$, sebab $\sum_{n=1}^{\infty} \Psi\left(y_{n}^{(m)}\right)=\Psi(k) \leq 1$.

Akibatnya, untuk setiap $m \geq 1$, berlaku

$\sum_{n=1}^{\infty}\left|x_{n}^{(t)}-x_{n}^{(p)}\right|\left|y_{n}^{(m)}\right|<\varepsilon$

$\Leftrightarrow\left|x_{m}^{(t)}-x_{m}^{(p)}\right||k|<\varepsilon \Leftrightarrow\left|x_{m}^{(t)}-x_{m}^{(p)}\right|<\frac{\varepsilon}{k}$,

untuk setiap $t, p \geq t_{0}$.

Dengan kata lain, untuk setiap $m \geq 1$, $\left(x_{m}^{(t)}\right)$ merupakan barisan Cauchy di dalam $\ell_{1}$. Karena $\ell_{1}$ lengkap, maka ada $x_{m} \in \mathfrak{R}$ sehingga $\lim _{t \rightarrow \infty} x_{m}^{(t)}=x_{m}$.

Selanjutnya, dibentuk $x=\left(x_{m}\right)$ dan ditunjukkan $x \in \ell_{\Phi}$.

Karena untuk setiap $y \in \tilde{\ell}_{\Psi}$ dengan $\sum_{n=1}^{\infty} \Psi\left(y_{n}\right) \leq 1$ dan untuk setiap $t \geq t_{0}$ berlaku

$\sum_{n=1}^{\infty}\left|x_{n}-x_{n}^{(t)}\right|\left|y_{n}\right|=\lim _{p \rightarrow \infty} \sum_{n=1}^{\infty}\left|x_{n}^{(p)}-x_{n}^{(t)}\right|\left|y_{n}\right| \leq \varepsilon$, maka $x^{(t)}-x \in \ell_{\Phi}$ dan karena $\left(\ell_{\Phi},\|.\|_{\Phi}\right)$ ruang linear berlaku $x=x^{(t)}-\left(x^{(t)}-x\right) \in \ell_{\Phi}$.

Di sisi lain, diperoleh bahwa $\lim _{t \rightarrow \infty}\left\|x^{(t)}-x\right\|_{\Phi}=0 . \quad$ Dengan kata lain, terbukti bahwa $\left(\ell_{\Phi},\|\|_{\Phi}\right)$ ruang Banach.
Berdasarkan Teorema 6 dan Teorema 7 dapat ditunjukkan bahwa $\left(\ell_{\Phi},\|\|_{\Phi}\right)$ merupakan ruang $\Phi$-lengkap sebagaimana yang dijelaskan dalam teorema berikut.

Teorema 9. Diberikan fungsi Orlicz $\Phi$ dan $\Psi$ komplemen fungsi $\Phi$. Ruang barisan Orlicz $\left(\ell_{\Phi},\|\|_{\Phi}\right)$ merupakan ruang $\Phi$-lengkap.

Bukti. Diambil sebarang barisan $\Phi$ Cauchy $\left(x^{(t)}\right) \subseteq \ell_{\Phi}$. Menurut Teorema 7, $\left(x^{(t)}\right)$ merupakan barisan Cauchy di dalam $\left(\ell_{\Phi},\|\|_{\Phi}\right)$. Karena $\left(\ell_{\Phi},\|\cdot\|_{\Phi}\right)$ ruang Banach, maka $\left(x^{(t)}\right)$ konvergen, katakan ke $x \in \ell_{\Phi}$. Menurut Teorema 6, diperoleh bahwa $\left(x^{(t)}\right) \Phi$-konvergen ke x. Dengan kata lain, $\left(\ell_{\Phi},\|\|_{\Phi}\right)$ merupakan ruang $\Phi$-lengkap. $\square$

\section{Kesimpulan}

Fungsi Orlicz $\Phi$ merupakan fungsi dari $\mathfrak{R}$ ke $\mathfrak{R}^{+}$dengan syaratsyarat tertentu. Ruang $X_{\Phi} \subset \omega$ merupakan ruang barisan yang dibangkitkan oleh fungsi Orlicz $\Phi$. Salah satu contoh dari ruang $X_{\Phi} \subset \omega$ adalah ruang $\ell_{\Phi}$ dengan definisi

$$
\ell_{\Phi}=\left\{x=\left(x_{n}\right): \sum_{n=1}^{\infty}\left|x_{n} y_{n}\right|<\infty, \forall y \in \tilde{\ell}_{\Psi}\right\}
$$


yang merupakan ruang bernorma terhadap norma Orlicz

$\|x\|_{\Phi}=\sup \left\{\sum_{n=1}^{\infty}\left|x_{n} y_{n}\right|: y \in \tilde{\ell}_{\Psi}, \sum_{n=1}^{\infty} \Psi\left(y_{n}\right) \leq 1\right\}$. Pada ruang $X_{\Phi}$ berlaku bahwa setiap barisan $\Phi$-konvergen merupakan barisan $\Phi$-Cauchy untuk $\Phi$ fungsi konveks dan memenuhi kondisi- $\Delta_{2}$. Akan tetapi, konversnya belum tentu berlaku di dalam $X_{\Phi}$. Selanjutnya, di dalam ruang $\left(\ell_{\Phi},\|\|_{\Phi}\right)$ berlaku bahwa setiap barisan $\Phi$-Cauchy merupakan barisan $\Phi$-konvergen. Selain itu, berlaku juga bahwa barisan $\Phi$ konvergen ekuivalen dengan konvergen (biasa). Begitu pula dengan barisan $\Phi$ Cauchy ternyata ekuivalen dengan barisan Cauchy. Karena adanya beberapa hubungan tersebut, disimpulkan bahwa ruang barisan $\left(\ell_{\Phi},\|\|_{\Phi}\right)$ merupakan ruang Banach sekaligus ruang $\Phi$-lengkap.

\section{Daftar Pustaka}

Alexopoulos, J., 2004, A brief introduction to $\mathrm{N}$-functions and Orlicz function spaces, Kent State University, Stark Campus.

Birnbaum, Z.W. and Orlicz, W., 1931, Über die Verallgemeinerung des Begriffes der zueinander konjugierten Funktionen, Studia Mathematica 3, pp. 1-67.
Kamthan, P.K. dan Gupta, M., 1981, Sequence Spaces and Series, Marcel Dekker, Inc., New York.

Khan, V.A., 2008, On A New Sequence Spaces Defined by Orlicz Function,

Commun.Fac.Sci.Univ.Ank.Serie s A1, Vol. 57, No. 2, pp.25-33.

Kolk, E., 2011, Topologies in Generalized Orlicz Sequence Spaces, Faculty of Science and Mathematics, University of Nis, Serbia.

Kreyszig, E., 1978, Introductory Functional Analysis with Applications, John Willey \& Sons, New York.

Lindberg, K.J., 1973, On Subspace of Orlicz Sequence Spaces, Studia Math.45:119-146.

Lindenstrauss, J. dan Tzafriri, L., 1973, On Orlicz Sequence Spaces:III, Israel J.Math.14:368-389.

Orlicz, W., 1932, Über eine gewisse Klasse von Räumen vom Typus B, Bull. Int. Acad. Polon. Sci. A 1932, 8/9, 207-220.

Rao, M.M. dan Ren, Z.D., 2002, Applications of Orlicz Spaces, Marcel Dekker Inc, New York.

Savas, E. dan Savas, R., 2004, Some Sequence Spaces Defined by Orlicz Function, Archivum Mathematicum (BRNO), Tomus 40, pp. 33-40. 